\title{
GENERALIZED SUMMING SEQUENCES AND THE MEAN ERGODIC THEOREM
}

\author{
JULIUS BLUM ${ }^{1}$ AND BENNETT EISENBERG ${ }^{2}$
}

\begin{abstract}
Conditions are found on a sequence of probability measures $\mu_{n}$ on a locally compact abelian group $G$ so that, for any strongly continuous unitary representation of $G, \int U_{\theta} f d \mu_{n}$ will converge to a $U$-invariant function. These conditions are applied in the case where the group is the integers.
\end{abstract}

Introduction. In a recent paper [3] Greenleaf discusses summing sequences in amenable locally compact groups. These are defined as sequences of compact sets $\left\{U_{n}\right\}$ in $G$ such that the following hold:

$$
\begin{gathered}
\operatorname{int}\left(U_{n+1}\right) \supseteq U_{n} \text { and } G=\bigcup_{n=1}^{\infty} U_{n} ; \\
\left|k U_{n} \Delta U_{n}\right| /\left|U_{n}\right| \rightarrow 0 \text { as } n \rightarrow \infty, \text { for every } k \in G,
\end{gathered}
$$

where $|E|$ is the left Haar measure of $E$.

These sequences have the property that if $G \times S \rightarrow S$ is a measurable action of $G$ on a measure space $(S, \mathscr{B}, \mu)$ such that $\mu$ is $G$-invariant, then, for $f$ in $L^{p},\left|U_{n}\right|^{-1} \int_{U_{n}} f(g s) d g$ converges in $L^{p}$ to a $G$-invariant $f^{*}$. Such results are proved using convexity and fixed point arguments.

In this paper attention is restricted to locally compact abelian groups thus allowing the full power of harmonic analysis to come into play. In $\$ 1$ more general summing sequences for the purpose of ergodic theorems as above are found and shown to include ordinary summing sequences. In $\$ 2$ more specific results are obtained on summing sequences when the group $G$ is the integers.

1. Generalized summing sequences. Let $U$ be a strongly continuous unitary representation of a locally compact abelian group $G$ on a Hilbert space $H$. If $\mu$ is a probability measure on $G, \int U_{o} f d \mu$ is defined weakly for each $f$ in $H$ so that, for all $h$ in $H$,

$$
\left(\int U_{g} f d \mu, h\right)=\int\left(U_{o} f, h\right) d \mu(g)
$$

Received by the editors May 15, 1973.

AMS (MOS) subject classifications (1970). Primary 28A65, 43A65, 43A25.

${ }^{1}$ Supported by NSF grant GP-25736.

${ }^{2}$ Supported by NSF grant GP-37771. 
A sequence of probability measures $\mu_{n}$ is called a generalized summing sequence if, for every unitary representation $U$ on $H$ and every $f$ in $H$, $\int U_{o} f d \mu_{n}$ converges in mean to an invariant element of $H$.

THEOREM 1. The following are equivalent:

(i) $\mu_{n}$ is a generalized summing sequence.

(ii) For every character $x$ on $G$ not equal to the identity the Fourier transforms $\hat{\mu}_{n}(x)$ converge to 0 .

(iii) $\mu_{n}$ considered as restrictions of measures on the Bohr compactification $\bar{G}$ of $G$ converge weakly to Haar measure on $\bar{G}$.

(iv) For every character $x$ of infinite order, the measures $\mu_{n}^{x}$ induced by $x$ on the unit circle in the complex plane converge weakly to normalized Lebesgue measure on the circle, and, for every character $x$ of order $m$, $m=0,1,2, \cdots$, the measures $\mu_{n}^{x}$ converge weakly to Haar measure on the mith roots of unity.

Proof. (ii) implies (i). Assume that $\hat{\mu}_{n}(x) \rightarrow 0$ for every character $x$ not the identity. Let $U$ be any unitary representation of $G$ and let $P f$ be the projection of $f$ on the subspace of $H$ invariant under $U$. Then $\int U_{o} P f d \mu_{n}(g)$ equals $P f$ for all $n$ and hence converges to $P f$.

$$
\begin{aligned}
\mid \int U_{g}(f-P f) d \mu_{n} \|^{2} & =\left(\int U_{g}(f-P f) d \mu_{n}, \int U_{h}(f-P f) d \mu_{n}\right) \\
& =\iint\left(U_{g}(f-P f), U_{h}(f-P f)\right) d \mu_{n}(g) d \mu_{n}(h),
\end{aligned}
$$

by (2).

Denote $\left(U_{g}(f-P f), f-P f\right)$ by $R(g)$. Then by Stone's theorem, $R(g)=\int\langle g, x\rangle d F(x)$, where $d F(A)=\left(E_{A}(f-P f), f-P f\right)$ and $E_{A}$ is the spectral resolution of $U$ over the dual group $G$. Hence

$$
\begin{aligned}
\| \int U_{g}(f-P f) & d \mu_{n} \|^{2} \\
& =\int_{G} \int_{G} R\left(g h^{-1}\right) d \mu_{n}(g) d \mu_{n}(h) \\
& =\int_{G} \int_{G} \int_{\hat{G}}\left\langle g h^{-1}, x\right\rangle d F(x) d \mu_{n}(g) d \mu_{n}(h) \\
& =\int_{x \neq 1} \int_{G} \int_{G}\langle g, x\rangle\left\langle h^{-1}, x\right\rangle d \mu_{n}(g) d \mu_{n}(h) d F(x)+\int_{x=1} d F(x) \\
& =\int_{x \neq 1}\left|\int_{G}\langle g, x\rangle d \hat{\mu}_{n}(g)\right|^{2} d F(x)+d F(1) .
\end{aligned}
$$

By dominated convergence and assumption (ii) this converges to $d F(1)$. 
But $E_{1}$ is the projection on the subspace of $H$ invariant under $U$, and since $f-P f$ is orthogonal to this subspace, $d F(1)=0$. Thus $\int U_{g}(f-P f) d \mu_{n}$ converges to 0 in mean.

Putting the pieces together $\int U_{g} f d \mu_{n}=\int U_{g} P f d \mu_{n}+\int U_{g}(f-P f) d \mu_{n}$ converges to $P f$ in mean.

(i) implies (ii). Assume $\hat{\mu}_{n}(x)$ does not converge to 0 , where $x$ is not identically one on $G$. Consider the unitary representation, where $U_{g}$ is multiplication by $\langle g, x\rangle$ on some Hilbert space $H$. Since $\langle g, x\rangle$ is not identically one, $P f=0$ for all $f$ in $H$. But

$$
\begin{aligned}
\left\|\int U_{g} f d \mu_{n}(g)\right\|^{2} & =\iint\langle g, x\rangle\left\langle h^{-1}, x\right\rangle\|f\|^{2} d \mu_{n}(g) d \mu_{n}(h) \\
& =\left|\hat{\mu}_{n}(x)\right|^{2}\|f\|^{2},
\end{aligned}
$$

which does not converge to 0 .

(ii) is equivalent to (iii). Give $G$ the discrete topology and call this new topological group $G_{d}$. Then the dual of $G_{d}$ is compact, contains $G$ as a dense subset, and is called the Bohr compactification $G$ of $G$. The characters on $G$, namely the elements of $G_{d}$, are precisely the characters of $G$, namely the elements of $G$. By the Levy continuity theorem for probability measures on a compact group, $\mu_{n}$ converges to Haar measure $\mu$ weakly if and only if $\hat{\mu}_{n}(x) \rightarrow \hat{\mu}(x)$, for all $x$. But the Fourier transform of Haar measure on a compact group is 1 at $x=1$ and 0 otherwise.

(ii) is equivalent to (iv). If $\mu_{n}$ is a measure on $G$, then each character $x$ of $G$ induces a measure $\mu_{n}^{x}$ on the unit circle in the complex plane defined by $\mu_{n}^{x}(B)=\mu_{n}\left(x^{-1} B\right)$ where $B$ is a Borel set in the circle. $\mu_{n}^{x}$ has the property that, for every integer $u$,

$$
\int e^{i \lambda u} d \mu_{n}^{x}(\lambda)=\int\langle x, g\rangle^{u} d \mu_{n}(g) .
$$

If (iv) is true then $\int e^{i \lambda u} d \mu_{n}^{x}(\lambda)$ converges to 0 as $n$ approaches infinity for each $u$ not a multiple of the order of $x$. In particular, if $x \neq 1$, then

$$
\int e^{i \lambda} d \mu_{n}^{x}(\lambda)=\int\langle g, x\rangle d \mu_{n}(g) \rightarrow 0 .
$$

Hence (ii) follows.

Conversely, assume $\int\langle g, x\rangle d \mu_{n}(g) \rightarrow 0$ for every $x \neq 1$. If $x$ is of infinite order this implies that for every integer $u \neq 0, \int\langle g, x\rangle^{u} d \mu_{n}(g) \rightarrow 0$ since $x^{u}$ is another character not equal to the identity. Hence $\int e^{i \lambda u} d \mu_{n}^{x}(\lambda)$ converges to 0 for $u \neq 0$ and again by the Levy continuity theorem $\mu_{n}^{x}$ converges weakly to Haar measure on the circle. 
If $x$ is of order $m$, then $x^{m}=1$ and $\langle g, x\rangle^{m}=1$. If $u$ is not a multiple of $m$, then $\int\langle g, x\rangle^{u} d \mu_{n}(x)=\int e^{i \lambda u} d \mu_{n}^{x}(\lambda)$ converges to 0 . But if $\mu$ is Haar measure on the $m$ th roots of unity, then $\hat{\mu}(u)$ is 1 if $\mu$ is a multiple of $m$ and 0 otherwise. Hence $\mu_{n}^{x}$ converges weakly to $\mu$.

Note that there is no loss of generality in the theorem in saying $\int U_{g} f d \mu_{n}$ converges to $P f$ since if it converges to any invariant function $\bar{f}$, that function must be $P f$. For if $h$ is invariant

$$
(f-\bar{f}, h)=\left(U_{g}(f-\bar{f}), U_{o} h\right)=\left(U_{g} f-\bar{f}, h\right) .
$$

Hence $(f-\bar{f}, h)=\left(\int U_{g} f d \mu_{n}-\bar{f}, h\right) \rightarrow 0$. Thus $\bar{f}=P f$.

Now assume $\mu_{n}$ is of the form $\mu_{n}(A)=\mu\left(A \cap E_{n}\right) / \mu\left(E_{n}\right)$, where $\mu$ is Haar measure on the group. We then say $\mu_{n}$ corresponds to $E_{n}$.

COROLlaRY 1. If $\mu_{n}$ corresponding to $E_{n}$ is a generalized summing sequence for $G$, then the sets $E_{n}$ cannot all lie in a proper closed subgroup $H$ of $G$.

Proof. $G / H$ is a nontrivial locally compact group. Hence there exists a character $x$ on $G / H$ not identically one. For if the dual group consisted of the identity alone, then by the duality theorem the original group would have to consist of the identity alone. This character induces a character on $G$ which is one on $H$. If $E_{n} \subset H$,

$$
\mu_{n}^{x}(1)=\mu\left(H \cap E_{n}\right) / \mu\left(E_{n}\right)=1 .
$$

Therefore $\mu_{n}^{x}$ does not converge weakly to Haar measure on the circle or on the $m$ th roots of unity for any $m \neq 1$. The corollary follows from (iv).

EXAMPLE. At first it appears that if $E_{n}=\{2,4, \cdots, 2 n\}$ then the corresponding $\mu_{n}$ form a generalized summing sequence for the integers. It is true that for any $N^{-1} \sum_{1}^{N} T^{2 n} f$ converges, by considering von Neumann's theorem applied to $T^{2}$. But the limit is invariant under $T^{2}$, not necessarily $T$. For example, if $T f=-f$ then $N^{-1} \sum_{i}^{N} T^{2 n} f=f$, which is invariant under $T^{2}=1$, but not $T$.

COROLlaRY 2. If $E_{n}$ is a sequence of sets such that, for any $g$ in $G$,

$$
\lim _{n} \frac{\mu\left(E_{n} \cap g E_{n}\right)}{\mu\left(E_{n}\right)}=1,
$$

then the corresponding $\mu_{n}$ form a generalized summing sequence for $G$.

Proof. Take any character $x$ not equal to the identity. Then there exists a $g_{0}$ such that $\left\langle g_{0}, x\right\rangle \neq 1$.

$$
\frac{\left\langle g_{0}, x\right\rangle}{\mu\left(E_{n}\right)} \int_{E_{n}}\langle g, x\rangle d \mu(g)=\frac{1}{\mu\left(E_{n}\right)} \int_{g_{0} E_{n}}\langle g, x\rangle d \mu(g)
$$


and by the assumption on $E_{n}$ this last expression is close to

$$
\left(\mu\left(E_{n}\right)\right)^{-1} \int_{E_{n}}\langle g, x\rangle d \mu(g)
$$

if $n$ is large enough. Since $\left\langle g_{0}, x\right\rangle$ is not one, it follows that if $n$ is large enough then $\left(\mu\left(E_{n}\right)\right)^{-1} \int_{E_{n}}\langle g, x\rangle d \mu(g)$ is arbitrarily close to zero. The corollary follows from (ii).

$\mu_{n}$ corresponding to $E_{n}$ satisfying the condition of Corollary 2 are essentially a summing sequence in that $\left|g E_{n} \Delta E_{n}\right| /\left|E_{n}\right| \rightarrow 0$ for every $g$ in $G$. But it is not necessary that $\operatorname{int}\left(E_{n+1}\right) \supset E_{n}$, that $E_{n}$ be compact, or that $\bigcup_{n=1}^{\infty} E_{n}=G$.

2. Generalized summing sequences when $G=Z$. In the case where $G=Z$ we will restrict attention to the case where $\mu_{n}$ corresponds to $E_{n}$ and $E_{n}=\left\{a_{1}, \cdots, a_{n}\right\}$, where $a_{n}$ is an increasing sequence of integers.

A few definitions are needed. A sequence of numbers $x_{j}$ is uniformly distributed on the circle if for every interval $I$, the proportion of the $x_{j}$ in $I$, for $j$ less than $N$, approaches the Haar measure of $I$ as $N$ approaches infinity. A sequence of numbers $x_{j}$ is uniformly distributed on the $m$ th roots of unity if the proportion of $x_{j}$ equal to each of these roots approaches $1 / m$. Note that weak convergence of probability measures $\mu_{n}$ to Haar measure on the circle is equivalent to convergence of $\mu_{n}(I)$ to the normalized length of $I$ for each interval $I$.

COROLlaRY 3. $N^{-1} \sum_{1}^{N} T^{n_{j}}$ converges strongly to the projection on the invariant subspace of $T$ for every unitary operator $T$ if and only if, for every $t$ in $[0,2 \pi], t$ not of the form $2 \pi n / m$, where $m$ and $n$ are integers, $\exp \left(\right.$ itn $\left._{j}\right)$ is uniformly distributed on the circle and for every $t$ such that $\exp (i t)$ is a primitive mth root of unity, $\exp \left(\right.$ itn $\left._{j}\right)$ is uniformly distributed on the mth roots of unity.

Proof. Clearly, $t$ of the first kind correspond to characters of infinite order and $t$ of the second kind to characters of order $m$. The measures $\mu_{n}$ involved in the corollary give measure $1 / n$ to the integers $n_{j}$ for $j=1$, $2, \cdots, N$ and measure zero to the rest of the integers. The induced measures on the circle $\mu_{\mathrm{N}}^{t}$ give measure to an interval $I$ equal to the proportion of the numbers $\exp \left(\right.$ itn $\left._{j}\right)$ falling in $I$ for $j$ less than or equal to $N$. The corollary now follows by noting that to say that $\exp \left(i t n_{j}\right)$ is uniformly distributed on the circle is equivalent to saying $\mu_{N}^{t}$ converges weakly to Haar measure on the circle and to say $\mu_{N}^{t}$ converges weakly to Haar measure on the $m$ th roots of unity is equivalent to saying $\exp \left(i t n_{j}\right)$ is uniformly distributed on the $m$ th roots of unity.

For more detailed results along this line see Blum and Mizel [2]. 
COROLLARY 4. If $\mu_{n}$ is a generalized summing sequence on $Z$ then for each subgroup $H=\{0, \pm m, \pm 2 m, \cdots\}, \lim \mu_{n}(g H)=m^{-1}$ for each coset of $H$.

Proof. Consider the character $x$ which takes $k$ into $\exp (2 \pi i k / m)$. Then $\mu_{n}^{x}(\{\exp (2 \pi i k / m)\})=\mu_{n}(k H)$ must approach $m^{-1}$.

Using Corollary 4 and some number theoretic arguments (see [1]) it can be shown that if $\mu_{n}$ is based on the following sequences of integers then $\mu_{n}$ is not a generalized summing sequence: $a_{n}=n !, a_{n}=k^{n}, a=n^{k}$ for $k$ an integer greater than one, and $a_{n}=p_{n}$ the $n$th prime. On the other hand, there do exist generalized summing sequences based on sequences of integers having density zero. For example, this is true if $a_{n}$ is the $n$th smallest integer in the set $m !+k, k=1$ to $m, m=1$ to infinity. This sequence has arbitrarily long consecutive sequences of integers spaced so as to give the whole sequence density zero. We see that this sequence is in fact an ordinary summing sequence.

Finally we give a sequence $\mu_{n}$ which is a generalized summing sequence, but not an ordinary summing sequence. Consider $\mu_{n}$ based on

$$
1,4,6,9,11,13,16,18,20, \cdots, n^{2}, n^{2}+2, \cdots, n^{2}+2(n-1),(n+1)^{2}, \cdots
$$

consisting of an odd, two consecutive evens, three consecutive odds, etc. but no two consecutive integers. Thus $\left(1+E_{n}\right) \Delta E_{n} \supset E_{n}$ and $\mu_{n}$ is not a summing sequence. Nevertheless, it is easy to see $\mu_{n}$ satisfies part (ii) of Theorem 1. Namely, if $\theta \neq 0$ or $\pi$ then

$$
\begin{gathered}
\left|\exp \left(i n^{2} \theta\right)+\exp \left(i\left(n^{2}+2\right)\right)+\cdots+\exp \left(i\left(n^{2}+2(n-1)\right) \theta\right)\right| \\
\leqq 2 /(1-\exp (2 i \theta))
\end{gathered}
$$

independently of $n$ and for $m=n(n+1) / 2$,

$$
\left|\mu_{m}(\theta)\right| \leqq 2 n /|1-\exp (2 i \theta)|\left(\frac{1}{2} n(n+1)\right) \rightarrow 0 .
$$

It easily follows $\mu_{n}(\theta) \rightarrow 0$. Moreover, if $\theta=\pi, \mu_{m}(\pi) \leqq 2 / n(n+1)$ and it easily follows $\mu_{n}(\pi) \rightarrow 0$.

It is an open question as to whether the pointwise ergodic theorem holds for generalized summing sequences even in the case $G=Z$.

\section{REFERENCES}

1. J. R. Blum, B. Eisenberg and L. S. Hahn, Ergodic theory and the measure of sets in the Bohr group, Acta Sci. Math. (Szeged). 34 (1973), 17-34. 
2. J. R. Blum and V. Mizel, On a theorem of Weyl and the ergodic theorem, Z. Wahrscheinlichkeitstheorie und Verw. Gebiete 20 (1971), 193-198.

3. Frederick P. Greenleaf, Ergodic theorems and the construction of summing sequences in amenable locally compact groups, Comm. Pure Appl. Math. 26 (1973), 29-46.

Department of Mathematics and Statistics, University of New Mexico, Albuquerque, New MeXico 87131

Department of Mathematics, Lehigh University, Bethlehem, Pennsyluania 18015 